\title{
BEHAVIOR OF SOLUTIONS TO ELLIPTIC EQUATIONS WITH NON-POWER NONLINEARITIES IN UNBOUNDED DOMAINS
}

\author{
R.KH. KARIMOV, L.M. KOZHEVNIKOVA, A.A. KHADZHI
}

\begin{abstract}
We establish estimates characterizing the decay rate as $|x| \rightarrow \infty$ of solutions to the Dirichlet problems in unbounded domains for a certain class of elliptic equations with non-power nonlinearities.
\end{abstract}

Keywords: anisotropic elliptic equations, non-power nonlinearity, Sobolev-Orlicz space, unbounded domain.

Mathematics Subject Classification: 35J62

\section{INTRODUCTION}

Let $\Omega$ be an arbitrary unbounded domain of the space $\mathbb{R}_{n}=\left\{x=\left(x_{1}, x_{2}, \ldots, x_{n}\right)\right\}, \Omega \subset \mathbb{R}_{n}$, $n \geqslant 2$. We consider the Dirichlet problem for quasilinear anisotropic second order elliptic equations

$$
\begin{gathered}
\sum_{\alpha=1}^{n}\left(a_{\alpha}(x, u, \nabla u)\right)_{x_{\alpha}}-a_{0}(x, u, \nabla u)=0, \quad x \in \Omega ; \\
\left.u\right|_{\partial \Omega}=0 .
\end{gathered}
$$

We assume that the functions $a_{\alpha}\left(x, s_{0}, s\right), \alpha=0, \ldots, n$, are measurable in $x \in \Omega$ for $\mathbf{s}=$ $\left(s_{0}, s\right)=\left(s_{0}, s_{1}, \ldots, s_{n}\right) \in \mathbb{R}_{n+1}$ and continuous in $\mathbf{s} \in \mathbb{R}_{n+1}$ for almost each $x \in \Omega$. Suppose that there exist positive numbers $\widehat{a}, \widehat{A}$ and measurable nonnegative functions $\psi(x), \Psi(x)$ such that the inequalities

$$
\begin{aligned}
& \sum_{\alpha=0}^{n} a_{\alpha}\left(x, s_{0}, s\right) s_{\alpha} \geqslant \bar{a} \sum_{\alpha=0}^{n} B_{\alpha}\left(s_{\alpha}\right)-\psi(x) ; \\
& \sum_{\alpha=0}^{n} \bar{B}_{\alpha}\left(a_{\alpha}\left(x, s_{0}, s\right)\right) \leqslant \widehat{A} \sum_{\alpha=0}^{n} B_{\alpha}\left(s_{\alpha}\right)+\Psi(x) \\
& \sum_{\alpha=0}^{n}\left(a_{\alpha}\left(x, s_{0}, s\right)-a_{\alpha}\left(x, t_{0}, t\right)\right)\left(s_{\alpha}-t_{\alpha}\right)>0
\end{aligned}
$$

hold true for almost each $x \in \Omega$ and $\mathbf{s}=\left(s_{0}, s\right), \mathbf{t}=\left(t_{0}, t\right) \in \mathbb{R}_{n+1}, \mathbf{s} \neq \mathbf{t}$.

Here $B_{0}(z), B_{1}(z), \ldots, B_{n}(z)$ are $N$-functions satisfying the $\Delta_{2}$-condition and $\bar{B}_{0}(z), \bar{B}_{1}(z)$, $\ldots, \bar{B}_{n}(z)$ are dual functions, see Section 1 .

R.Kh. Karimov, L.M. Kozhevnikova, A.A. Khadzhi, Behavior of solutions to elliptic equaTIONS WITH NON-POWER NONLINEARITIES IN UNBOUNDED DOMAINS.

(C) Karimov R.Kh., Kozhevnikova L.M., Khadzhi A.A. 2016.

The research is supported by the grant of the Russian Science Foundation (project no. 16-31-50034\16).

Submitted November 19, 2015. 
As an example we can consider the equation

$$
\sum_{\alpha=1}^{n}\left(B_{\alpha}^{\prime}\left(u_{x_{\alpha}}\right)+f_{\alpha}(x)\right)_{x_{\alpha}}-B_{0}^{\prime}(u)-f_{0}(x)=0
$$

with continuously differentiable $N$-functions $B_{0}(z), B_{1}(z), \ldots, B_{n}(z)$ (see Lemma 4).

Starting from the 70s of the last century (see [1]- [4]) and till the present, the qualitative properties are intensively studied for the solutions to ellptic equations with non-power nonlinearities both of second and higher orders. The solutions to boundary values problems for the equations of form (0.1) with the functions $a_{0}(x, \mathbf{s}), a_{1}(x, \mathbf{s}), \ldots, a_{n}(x, \mathbf{s})$ having not only polynomial growth in the variables $s_{0}, s_{1}, \ldots, s_{n}$ were considered mostly in bounded domains. For example, in work [5], the Dirichlet problem in a bounded domain $\Omega$ was studied for a nonlinear elliptic equation with a vector function $\mathbf{a}(x, s)=\left(a_{1}(x, s), \ldots, a_{n}(x, s)\right)$ satisfying non-standard growth conditions described in terms of $N$-depending on $x$. The existence the renormalized solution was proved, while under the strong monotonicity condition the uniqueness was established.

Boundary value problems in unbounded domains for quasilinear elliptic equations with power nonlinearities were also studied in works [6], [7. It should be noted that a solution to an elliptic problem in an unbounded domain with non-summable data belongs to the corresponding space of locallly summable functions. As a rule, to ensure the uniqueness of the solution of the corresponding boundary value problem in an unbounded domain, one has to impose a restriction for the growth of the solution at the infinity, while for the existence of a solution in the selected class one usually needs the restrictions for the growth of the input data [8].

In 1984 , by the semi-linear equation

$$
-\Delta u+|u|^{p_{0}-2} u=f(x), \quad x \in \mathbb{R}_{n}, \quad p_{0}>2,
$$

H. Brezis showed [9] that there exist elliptic equations for which there exist unique solutions to the boundary value problems with no conditions for their behavior and the growth of the input data at the infinity. Namely, H. Brezis established the existence and the uniqueness of the solution $u \in L_{p_{0}-1, \text { loc }}\left(\mathbb{R}_{n}\right)$ as $f \in L_{1, \text { loc }}\left(\mathbb{R}_{n}\right)$. The results by $H$. Brezis were generalized for the equations of higher order by F. Bernis [10].

In work [11], J.I. Diaz and O.A. Oleinik employed the energy integral method and established apriori estimates for a solution to prove the unique solvability for the boundary value problem with homogeneous boundary condition of first and second type (in particular, for the Dirichlet and Neumann problems) for semi-linear equations with variable coefficients

$$
-\sum_{i, j=1}^{n}\left(a_{i j}(x) u_{x_{j}}\right)_{x_{i}}+a_{0}(x)|u|^{p_{0}-2} u=f(x), \quad x \in \Omega, \quad p_{0}>2,
$$

$a_{i j}(x) \in L_{\infty, \mathrm{loc}}(\Omega), a_{0}(x) \in L_{1, \mathrm{loc}}(\Omega), a_{0}(x) \geqslant a_{0}>0$ with no conditions at the infinity. Moreover, in [11] the authors studied the asymptotic behavior at infinifty for the solution to equation (0.7). Under the condition $f(x)=0, x \in \Omega \backslash \bar{\Omega}\left(r_{0}\right), \bar{\Omega}\left(r_{0}\right)=\left\{x \in \Omega|| x \mid \leqslant r_{0}\right\}, r_{0}>0$, for a solution to equation 0.7 the estimate

$$
|u(x)| \leqslant C_{1}|x|^{-2 /\left(p_{0}-2\right)}, \quad x \in \Omega \backslash \bar{\Omega}\left(r_{0}\right) .
$$

was obtained. Under an additional restriction for the geometry of the unbounded domain $\Omega$ the inequality

was established.

$$
|u(x)| \leqslant C_{2} e^{-\alpha|x|}, \quad x \in \Omega \backslash \bar{\Omega}\left(r_{0}\right), \quad \alpha>0,
$$

In work [12], M.M. Bokalo and E.V. Domanska studied boundary value problems in unbounded domains for elliptic anisotropic equations with variable nonlinearity exponents. At 
that, the well-posedness of the boundary value problems was proved with no restrictions for the growth of the solutions and data at the infinity.

The authors of the present work succeeded to select some class of elliptic equations having not only power nonlinearities and to obtain the results close to the cited above. For instance, in work [13] by L.M. Kozhevnikova, A.A. Khadzhi, the solvability of the Dirichlet problem in unbounded domains was established with no restrictions for the growth of the data at infinity. Under additional restrictions for the stucture of the equation, in [14 the uniqueness of the solution to problem $0.1,0.2$ was proved with no restrictions for the growth of the solution at the infinity.

Here we obtain the estimates characterizing the behavior as $|x| \rightarrow \infty$ of the solution to problem (0.1), (0.2) in unbounded domains $\Omega$. A power estimate was established for the solutions to anisotropic equations in arbitrary unbounded domains (Theorem 2). For "nonwide" unbounded domains we obtained an exponential estimates for the solutions to isotropic equations (Theorem 3).

\section{N-FunCtions AND Sobolev-Orlicz SPACES}

Let us provide necessary information from the theory of $N$-functions and Sobolev-Orlicz spaces [15]. A non-negative continuous convex function $M(z), z \in \mathbb{R}$, is called $N$-function if it is even and $\lim _{z \rightarrow 0} M(z) / z=0, \lim _{z \rightarrow \infty} M(z) / z=\infty$. We note that $M(\epsilon z) \leqslant \epsilon M(z)$ as $0<\epsilon \leqslant 1$. For an $N$-function $M(z)$ the integral representation $M(z)=\int_{0}^{|z|} m(\theta) d \theta$ holds, where $m(\theta)$ is positive as $\theta>0$, is non-decreasing and right continuous as $\theta \geqslant 0$ and $m(0)=0, \lim _{\theta \rightarrow \infty} m(\theta)=\infty$.

For an $N$-function $M(z)$ and its dual $N$-function

$$
\bar{M}(z)=\sup _{y \geqslant 0}(y|z|-M(y))
$$

the Young inequality

$$
|z y| \leqslant M(z)+\bar{M}(y), \quad z, y \in \mathbb{R}
$$

is satisfied [15, Ch. I, Sect. 2, Ineq. (2.6)].

Given $N$-functions $P(z), M(z)$, we write $P(z) \prec M(z)$ if there exist numbers $l>0, z_{0} \geqslant 0$ such that

$$
P(z) \leqslant M(l z), \quad|z| \geqslant z_{0} .
$$

$N$-functions $P(z), M(z)$ are called comparable if one of the relations $P(z) \prec M(z)$ or $M(z) \prec$ $P(z)$ is satisfied. $N$-function $P(z)$ and $M(z)$ are called equivalent if $P(z) \prec M(z)$ and $M(z) \prec$ $P(z)$.

An $N$-function $P(z)$ grows slowly than an $N$-function $M(z)(P(z) \prec \prec M(z))$ if

$$
\lim _{z \rightarrow \infty} P(z) / M(l z)=0
$$

for each number $l>0$.

An $N$-function $M(z)$ satisfies the $\Delta_{2}$-condition for large $z$ if there exist numbers $c>0, z_{0} \geqslant 0$ such that $M(2 z) \leqslant c M(z)$ for all $|z| \geqslant z_{0}$. The $\Delta_{2}$-condition is equivalent to the inequality

$$
M(l z) \leqslant c(l) M(z)
$$

as $|z| \geqslant z_{0}$, where $l$ is an arbitrary number larger than one, $c(l)>0$.

In each class of equivalent $N$-function obeying the $\Delta_{2}$-condition there exist $N$-functions satisfying inequality 1.2 for all $z$. Hereafter we assume that the $\Delta_{2}$-condition is satisfied for the considered $N$-functions for all values $z \in \mathbb{R}$ (i.e., $z_{0}=0$ ).

Due to the convexity and estimate $(1.2)$, the $N$-function $M(z)$ satisfies the inequality

$$
M(y+z) \leqslant c M(z)+c M(y), \quad z, y \in \mathbb{R} .
$$


Let $Q$ be an arbitrary domain in the space $\mathbb{R}_{n}$. The Orlicz class $K_{M}(Q)$ associated with an $N$-function $M(z)$ is the set of functions $v$ measurable in $Q$ such that

$$
\int_{Q} M(v(x)) d x<\infty .
$$

The Orlicz space $L_{M}(Q)$ is the linear span of $K_{M}(Q)$. we shall consider the Orlicz space $L_{M}(Q)$ with the Luxembourg norm

$$
\|v\|_{L_{M}(Q)}=\|v\|_{M, Q}=\inf \left\{k>0 \mid \int_{Q} M(v(x) / k) d x \leqslant 1\right\} .
$$

The Orlicz class $K_{M}(Q)$ coincides with the Orlicz space $L_{M}(Q)$ if and only if $M(z)$ satisfies the $\Delta_{2}$-condition [15, Ch. II, Sect. 8, Thm. 8.2].

Given a function $v \in L_{M}(Q)$, the estimate

$$
\|v\|_{M, Q} \leqslant \int_{Q} M(v) d x+1
$$

hold true [15, Ch. II, Sect. 9, Ineq. (9.12)]. Functions $u \in L_{M}(Q), v \in L_{\bar{M}}(Q)$ saitsfy the Hölder inequality [15, Ch. II, Sect. 9, Ineq. (9.24), (9.27)]:

$$
\left|\int_{Q} u(x) v(x) d x\right| \leqslant 2\|u\|_{M, Q}\|v\|_{\bar{M}, Q} .
$$

Given $N$-functions $B_{1}(z), \ldots, B_{n}(z)$, we introduce the Sobolev-Orlicz space $\stackrel{\circ}{H}_{B}^{1}(Q)$ as the completion of $C_{0}^{\infty}(Q)$ w.r.t. the norm

$$
\|v\|_{H_{B}^{1}(Q)}=\sum_{\alpha=1}^{n}\left\|v_{x_{\alpha}}\right\|_{B_{\alpha}, Q} .
$$

The norms in the spaces $L_{1}(Q), L_{\infty}(Q)$ are denoted by $\|\cdot\|_{1, Q},\|\cdot\|_{\infty, Q}$, respectively.

We let

$$
h(t)=t^{-1 / n}\left(\prod_{\alpha=1}^{n} B_{\alpha}^{-1}(t)\right)^{1 / n}
$$

and assume that the integral $\int_{0}^{1} h(t) / t d t$ converges. Then we can define an $N$-function $B^{*}(z)$ by the formula

$$
\left(B^{*}\right)^{-1}(z)=\int_{0}^{|z|} h(t) / t d t
$$

We provide the embedding theorem by A.G. Korolev [16] proven for bounded domains $Q$.

Lemma 1. Let $v \in \stackrel{\circ}{H}_{B}^{1}(Q)$.

1) If

$$
\int_{1}^{\infty} h(t) / t d t=\infty
$$

then $\stackrel{\circ}{H}_{B}^{1}(Q) \subset L_{B^{*}}(Q)$ and

$$
\|v\|_{B^{*}, Q} \leqslant A_{1}\|v\|_{\stackrel{\circ}{B}_{B}^{1}(Q)} ;
$$


2) If

$$
\int_{1}^{\infty} h(t) / t d t<\infty
$$

then $\stackrel{\circ}{H}_{B}^{1}(Q) \subset L_{\infty}(Q)$ and

$$
\|v\|_{\infty, Q} \leqslant A_{2}\|v\|_{\stackrel{\circ}{B}_{B}^{1}(Q)} .
$$

Here $A_{1}=\frac{n-1}{n}, A_{2}=\int_{0}^{\infty} \frac{h(t)}{t} d t$.

Thanks to the $\Delta_{2}$-condition, the convergence in the norm is equivalent to the mean convergence [15, Ch. II, Sect. 9, Thm. 9.4]. Moreover, in [17] the following lemma was proved.

Lemma 2. If an $N$-function $M(z)$ satisfies the $\Delta_{2}$-condition, $v(x), v^{i}(x) \in L_{M}(Q), i=$ $1,2, \ldots, v^{i}(x) \rightarrow v(x)$ in $L_{M}(Q)$, then

$$
\int_{Q}\left|M\left(v^{i}\right)-M(v)\right| d x \rightarrow 0, \quad i \rightarrow \infty .
$$

\section{Formulation OF THEOREMS}

Assume that $N$-functions $B_{0}(z), B_{1}(z), \ldots, B_{n}(z)$ and their dual $N$-functions $\bar{B}_{0}(z), \bar{B}_{1}(z)$, $\ldots, \bar{B}_{n}(z)$ satisfy the $\Delta_{2}$-condition.

By $\mathbf{L}_{\overline{\mathbf{B}}}(\Omega)$ we denote the space $L_{\bar{B}_{0}}(\Omega) \times L_{\bar{B}_{1}}(\Omega) \times \ldots \times L_{\bar{B}_{n}}(\Omega)$ with the norm

$$
\|\mathbf{g}\|_{\mathbf{L}_{\overline{\mathbf{B}}}(\Omega)}=\left\|g_{0}\right\|_{\bar{B}_{0}, \Omega}+\left\|g_{1}\right\|_{\bar{B}_{1}, \Omega}+\ldots+\left\|g_{n}\right\|_{\bar{B}_{n}, \Omega}, \quad \mathbf{g}=\left(g_{0}, g_{1}, \ldots, g_{n}\right) \in \mathbf{L}_{\overline{\mathbf{B}}}(\Omega) .
$$

We introduce a Sobolev-Orlicz space $\stackrel{\circ}{W}_{\mathbf{B}}^{1}(\Omega)$ as the completion of the space $C_{0}^{\infty}(\Omega)$ w.r.t. the norm

$$
\|v\|_{\stackrel{\circ}{\mathbf{B}}_{\mathbf{B}}(\Omega)}=\|v\|_{B_{0}, \Omega}+\|v\|_{\stackrel{\circ}{B}_{B}^{1}(\Omega)} .
$$

If condition (1.6) is satisfied, we assume that

$$
B_{0}(z) \prec B^{*}(z),
$$

while in the case of $(1.7), B_{0}(z)$ is an arbitrary $N$-function.

We define $L_{1, \text { loc }}(\bar{\Omega}), \stackrel{\circ}{\text { B loc }}^{1}(\bar{\Omega})$ as the spaces consisting of the functions $v(x)$ defined in $\Omega$ and such that for each bounded $Q \subset \Omega$ there exists a function in the space $L_{1}(\Omega), \stackrel{\circ}{W}_{\mathbf{B}}^{1}(\Omega)$, respectively, coinciding with the function $v(x)$ in $Q$. For nonnegative functions we assume that $\psi(x), \Psi(x) \in L_{1, \text { loc }}(\bar{\Omega})$. In the same way we define the space $\mathbf{L}_{\overline{\mathbf{B}}, \text { loc }}(\bar{\Omega})$.

We define the operator $\mathbf{B}: \stackrel{\circ}{W}_{\mathbf{B}, \text { loc }}^{1}(\bar{\Omega}) \rightarrow L_{1, \text { loc }}(\bar{\Omega})$ by the formula

$$
\mathbf{B}(v)=B_{0}(v)+\sum_{\alpha=1}^{n} B_{\alpha}\left(v_{x_{\alpha}}\right), \quad v \in \stackrel{\circ}{W}_{\mathbf{B}, \mathrm{loc}}^{1}(\bar{\Omega}) .
$$

We denote

$$
\mathbf{a}(x, \mathbf{s})=\left(a_{0}(x, \mathbf{s}), a_{1}(x, \mathbf{s}), \ldots, a_{n}(x, \mathbf{s})\right) .
$$

Employing (1.4), by condition (0.4) we get the estimate

$$
\begin{aligned}
\|\mathbf{a}(x, u, \nabla u)\|_{\mathbf{L}_{\overline{\mathbf{B}}}(Q)} & =\sum_{\alpha=0}^{n}\left\|a_{\alpha}(x, u, \nabla u)\right\|_{\bar{B}_{\alpha}, Q} \\
& \leqslant \sum_{\alpha=0}^{n} \int_{Q} \bar{B}_{\alpha}\left(a_{\alpha}(x, u, \nabla u)\right) d x+n+1 \leqslant \widehat{A}\|\mathbf{B}(u)\|_{1, Q}+\|\Psi\|_{1, Q}+n+1
\end{aligned}
$$


for $u \in \stackrel{\circ}{W}_{\mathbf{B}, \text { loc }}^{1}(\bar{\Omega})$ and each bounded $Q \subset \Omega$.

Given an element $\mathbf{a}(x, u, \nabla u) \in \mathbf{L}_{\overline{\mathbf{B}}, \text { loc }}(\bar{\Omega})$, for compactly supported $v(x) \in \stackrel{\circ}{W}_{\mathbf{B}}^{1}(\Omega)$ we introduce a functional $\mathbf{A}(u)$ by the identity:

$$
\langle\mathbf{A}(u), v\rangle=\int_{\Omega}\left(\sum_{\alpha=1}^{n} a_{\alpha} v_{x_{\alpha}}+a_{0} v\right) d x .
$$

Employing Hölder inequality $(1.5)$, for functions $u(x) \in \stackrel{\circ}{W}_{\mathbf{B}, \text { loc }}^{1}(\bar{\Omega}), v(x) \in \stackrel{\circ}{W}_{\mathbf{B}}^{1}(\Omega)(\operatorname{supp} v=$ $\left.\bar{Q}_{v}\right)$ we obtain the inequalities

$$
\begin{array}{r}
|\langle\mathbf{A}(u), v\rangle| \leqslant 2 \sum_{\alpha=1}^{n}\left\|a_{\alpha}\right\|_{\bar{B}_{\alpha}, Q_{v}}\left\|v_{x_{\alpha}}\right\|_{B_{\alpha}, Q_{v}}+2\left\|a_{0}\right\|_{\bar{B}_{0}, Q_{v}}\|v\|_{B_{0}, Q_{v}} \leqslant \\
\leqslant 2\|\mathbf{a}(x, u, \nabla u)\|_{\mathbf{L}_{\overline{\mathbf{B}}}\left(Q_{v}\right)}\|v\|_{\dot{W}_{\mathbf{B}}^{1}(\Omega)} .
\end{array}
$$

Thus, estimates 2.2), (2.4) imply the boundedness of the functional $\mathbf{A}(u)$ in the space of compactly supported functions in $\stackrel{\circ}{W}_{\mathbf{B}}^{1}(\Omega)$.

Definition 1. A generalized solution to problem $(0.1),(0.2)$ is a function $u(x) \in W_{\mathbf{B}, \text { loc }}^{1}(\bar{\Omega})$ satisfying the integral identity

$$
\langle\mathbf{A}(u), v\rangle=0
$$

for each compactly supported function $v(x) \in \stackrel{\circ}{W}_{\mathbf{B}}^{1}(\Omega)$.

We suppose that there exists $0<\epsilon<1$ such the conditions

$$
B_{\alpha}\left(z^{1+\epsilon}\right) \prec B_{0}(z), \quad \alpha=1,2, \ldots, n,
$$

hold true.

In work [13], the solvability of problem 0.1 , 0.2 was proven in arbitrary unbounded domains $\Omega$. Namely, the following theorem was established.

Theorem 1. Assume that conditions (0.3) - (0.5), 2.6) are satisfied. Then there exists a generalized solution $u(x)$ to problem $(0.1), 0.2$.

A power estimate for the decay rate of the solution was obtained under the condition that

$$
B_{\alpha}(z)=c_{\alpha}|z|^{p_{\alpha}}, \quad|z|<1, \quad p_{\alpha}>1, \quad c_{\alpha}>0, \quad \alpha=0,1, \ldots, n .
$$

We note that given an arbitrary $N$-function $\widetilde{B}(z)$, such $N$-function can be constructed easily:

$$
B(z)=\left\{\begin{array}{l}
\widetilde{B}(1)|z|^{p},|z|<1 ; \quad p=\frac{\widetilde{B}^{\prime}(1)}{\widetilde{B}(1)}>1 . \\
\widetilde{B}(z),|z| \geqslant 1,
\end{array}\right.
$$

At that, the functions $\widetilde{B}(z), B(z)$ are equivalent.

We assume that the exponents $p_{\alpha}, \alpha=1, \ldots, n$, are ordered: $p_{1} \geqslant p_{2} \geqslant \ldots \geqslant p_{n}$ and obey the conditions:

$$
p_{0}>p_{1}, \quad \sum_{\alpha=1}^{n} \frac{1}{p_{\alpha}}>1 .
$$

Then then numbers $q_{\alpha}=\frac{p_{0} p_{\alpha}}{p_{0}-p_{\alpha}}, \alpha=1, \ldots, n$, are ordered as well: $q_{1} \geqslant q_{2} \geqslant \ldots \geqslant q_{n}$. We assume that

$$
q_{n}>n
$$


Theorem 2. Assuem that conditions (0.3)-(0.5), (2.6) $-(2.8)$ are satisfied. Then there exists a positive number $\mathcal{M}_{1}$ such that a generalized solution to problem (0.1), (0.2) satisfies the estimate

$$
\|\mathbf{B}(u)\|_{1, \Omega(r / 2)} \leqslant \mathcal{M}_{1}\left(r^{n-q_{n}}+\|\psi+\Psi\|_{1, \Omega(r)}\right), \quad r \geqslant 1,
$$

where $\Omega(r)=\{x \in \Omega|| x \mid<r\}$.

For example, the assumption of Theorem 2 are satisfied for equation $(0.6)$ with the functions

$$
B_{\alpha}(z)=\left\{\begin{array}{l}
|z|^{p_{\alpha}},|z|<1 \\
|z|^{p_{\alpha}-1}(\ln |z|+1),|z| \geqslant 1
\end{array}\right.
$$

under an appropriate choice of $p_{\alpha}>2, \alpha=0,1, \ldots, n$ (see Example 1 ).

For unbounded domains located along a selected axis, in works [17, [18] the authors established exponential estimates for the decay rate of a solution to problem (0.1), (0.2) with compactly supported data in terms of a special geometric characteristics. Here we succeeded to obtain an exponential estimate for the isotropic case

$$
B_{\alpha}(z)=B(z), \quad \alpha=1,2, \ldots, n,
$$

for unbounded domains satisfying the only condition

$$
d(r)=\operatorname{diam} \gamma(r) \leqslant D, \quad D>0, \quad \gamma(r)=\{x \in \Omega|| x \mid=r\}, \quad r \geqslant r_{1} .
$$

Theorem 3. Assume that conditions (0.3)-(0.5), (2.6), (2.11), (2.12) hold true. Then there exist positive numbers $\kappa, \mathcal{M}_{2}, r_{0}$ such that a solution $u(x)$ to problem (0.1), (0.2) satisfies the estimate

for all $r \geqslant r_{0}$.

$$
\|\mathbf{B}(u)\|_{1, \Omega(r / 2)} \leqslant \mathcal{M}_{2}\left(\exp (-\kappa r) r^{n-1}+\|\psi+\Psi\|_{1, \Omega(2 r)}\right)
$$

It should be mentioned that estimates 2.10 , 2.13) obtained in this work are in an agreement with the results of paper [11].

\section{PRELIMINARIES}

Lemma 3. Assume that $N$-functions $B_{0}(z), B_{1}(z), \ldots, B_{n}(z)$ satisfy conditions (2.6), then

$$
B_{\alpha}(z) \prec \prec B_{0}(z), \quad \alpha=1,2, \ldots, n .
$$

For the proof of Lemma see [13, Rem. 6].

Lemma 4. If the functions $b_{\alpha}\left(s_{\alpha}\right)=B_{\alpha}^{\prime}\left(s_{\alpha}\right), s_{\alpha} \geqslant 0, \alpha=0,1, \ldots, n$, are continuous and strictly monotonous, $\mathbf{f}=\left(f_{0}, f_{1}, \ldots, f_{n}\right) \in \mathbf{L}_{\overline{\mathbf{B}}, \text { loc }}(\bar{\Omega})$, then the functions

$$
a_{\alpha}\left(x, s_{\alpha}\right)=B_{\alpha}^{\prime}\left(s_{\alpha}\right)+f_{\alpha}(x)=b_{\alpha}\left(\left|s_{\alpha}\right|\right) \operatorname{sign} s_{\alpha}+f_{\alpha}(x), \quad \alpha=0, \ldots, n,
$$

satisfy conditions $0.3-0.5$.

For the proof of the lemma see [13, Rem. 5].

Hereinafter in this section by $C_{i}$ we denote positive constants.

Lemma 5. Assume that $N$-functions $B_{0}(z), B_{1}(z), \ldots, B_{n}(z)$ satisy conditions (2.6), then for the $N$-functions $T_{\alpha}(z)=B_{\alpha}\left(\bar{M}_{\alpha}(z)\right),\left(M_{\alpha}(z)=B_{\alpha}^{-1}\left(B_{0}(z)\right)\right.$ there exist numbers $c>0$, $\tau \geqslant q_{n}$ such that the inequalities

$$
T_{\alpha}(z) \leqslant c|z|^{\tau}, \quad|z| \geqslant 1, \quad \alpha=1,2, \ldots, n,
$$

hold true.

For the proof of the lemma see [14, Lm. 3.3]. 
Lemma 6. Assume that $N$-functions $B_{0}(z), B_{1}(z), \ldots, B_{n}(z)$ satisfy conditions (2.7), (2.8), then for the $N$-functions $T_{\alpha}(z)=B_{\alpha}\left(\bar{M}_{\alpha}(z)\right)$ there exists a number $c>0$ such that the inequalities

$$
T_{\alpha}(z) \leqslant c|z|^{q_{\alpha}}, \quad|z| \leqslant 1, \quad \alpha=1,2, \ldots, n
$$

hold.

For the proof of the lemma see [14, Lm. 3.4].

Lemma 7. Let $\Sigma_{R, d}$ be a spherical segment of a diameter $d$ on the sphere of a radius $R$, $d \leqslant R / 8$, in the space $\mathbb{R}_{n}, n \geqslant 2$. If an $N$-function $B(z)$ satisfies the $\Delta_{2}$-condition, then there exists a constant $c(n)>0$ such that the function $v(x) \in C_{0}^{\infty}\left(\mathbb{R}_{n}\right),\left.v\right|_{\Sigma_{R, d}} \in C_{0}^{\infty}\left(\Sigma_{R, d}\right)$ satisfies the inequality

$$
\int_{\Sigma_{R, d}} B(v) d S \leqslant c \int_{\Sigma_{R, d}} B(d|\nabla v|) d S .
$$

For the proof of the lemma see [19].

\section{Proof of Theorem 2}

Proof. Let $\xi$ be an absolutely continuous nonnegative compactly supported function. Letting $v=\xi^{p} u, p \geqslant \tau$, in identity (2.5) (see Lemma 5), we obtain the inequality

$$
\begin{aligned}
& \int_{\Omega} \xi^{p}\left(\sum_{\alpha=1}^{n} a_{\alpha}(x, u, \nabla u) u_{x_{\alpha}}+a_{0}(x, u, \nabla u) u\right) d x \\
& \quad \leqslant p \sum_{\alpha=1}^{n} \int_{\Omega}\left|a_{\alpha}(x, u, \nabla u)\|u\| \xi_{x_{\alpha}}(x)\right| \xi^{p-1} d x=p \cdot J_{1} .
\end{aligned}
$$

Applying (1.1), for $\varepsilon \in(0,1)$ we get

$$
\begin{aligned}
J_{1} & \leqslant \sum_{\alpha=1}^{n} \int_{\Omega} \xi^{p}\left(\bar{B}_{\alpha}\left(\varepsilon a_{\alpha}(x, u, \nabla u)\right)+B_{\alpha}\left(\frac{u}{\varepsilon} \frac{\xi_{x_{\alpha}}}{\xi}\right)\right) d x \\
& \leqslant \sum_{\alpha=1}^{n} \int_{\Omega} \xi^{p} \varepsilon \bar{B}_{\alpha}\left(a_{\alpha}(x, u, \nabla u)\right) d x+\sum_{\alpha=1}^{n} \int_{\Omega} \xi^{p} B_{\alpha}\left(\frac{u}{\varepsilon} \frac{\xi_{x_{\alpha}}}{\xi}\right) d x=J_{11}+J_{12} .
\end{aligned}
$$

Let us estimate integral $J_{12}$. Since according to Lemma 3, relations 3.1 hold true, then the $N$-functions $B_{0}(z)=B_{\alpha}\left(M_{\alpha}(z)\right)$ can be represented as compositions of two $N$-functions $M_{\alpha}(z), B_{\alpha}(z), \alpha=1, \ldots, n$. Applying (1.1), 1.3), we establish that

$$
\begin{aligned}
J_{12} & \leqslant \sum_{\alpha=1}^{n} \int_{\Omega} \xi^{p} B_{\alpha}\left\{M_{\alpha}(\varepsilon u)+\bar{M}_{\alpha}\left(\frac{1}{\varepsilon^{2}} \frac{|\nabla \xi|}{\xi}\right)\right\} d x \\
& \leqslant \sum_{\alpha=1}^{n} \int_{\Omega} \xi^{p}\left(\varepsilon C_{1} B_{0}(u)+C_{1} B_{\alpha}\left(\bar{M}_{\alpha}\left(\frac{1}{\varepsilon^{2}} \frac{|\nabla \xi|}{\xi}\right)\right)\right) d x \\
& =C_{1}\left(\varepsilon n \int_{\Omega} \xi^{p} B_{0}(u) d x+J_{2}\right),
\end{aligned}
$$


where

$$
J_{2}=\sum_{\alpha=1}^{n} \int_{\Omega} \xi^{p} T_{\alpha}\left(\frac{1}{\varepsilon^{2}} \frac{|\nabla \xi|}{\xi}\right) d x, \quad T_{\alpha}(z)=B_{\alpha}\left(\bar{M}_{\alpha}(z)\right) .
$$

Combining (4.2), (4.3) and employing condition (0.4), we get

$$
\begin{aligned}
J_{1} \leqslant & \int_{\Omega} \xi^{p} \varepsilon\left(\widehat{A} \sum_{\alpha=1}^{n} B_{\alpha}\left(u_{x_{\alpha}}\right)+\left(C_{1} n+\widehat{A}\right) B_{0}(u)\right) d x \\
& +\int_{\Omega} \xi^{p} \Psi d x+C_{1} J_{2} \leqslant \varepsilon C_{2} \int_{\Omega} \xi^{p} \mathbf{B}(u) d x+\int_{\Omega} \xi^{p} \Psi d x+C_{1} J_{2} .
\end{aligned}
$$

Employing (0.3), by (4.1), 4.5) we obtain the estimate

$$
\bar{a} \int_{\Omega} \xi^{p} \mathbf{B}(u) d x \leqslant \varepsilon p C_{2} \int_{\Omega} \xi^{p} \mathbf{B}(u) d x+p \int_{\Omega} \xi^{p}\{\Psi+\psi\} d x+C_{1} p J_{2} .
$$

Choosing $\varepsilon$ sufficiently small, we have the inequality

$$
\left\|\xi^{p} \mathbf{B}(u)\right\|_{1} \leqslant C_{3} \int_{\Omega} \xi^{p}\{\Psi+\psi\} d x+C_{4} J_{2} .
$$

Let $r_{0}$ be an arbitrary positive number. We fix $r>r_{0}$ and consider the cut-off function $\xi(x)=\frac{1}{r}\left(r^{2}-|x|^{2}\right)$ as $|x|<r, \xi(x)=0$ as $|x| \geqslant r$. Let us justify the finiteness of the integral $J_{2}$. It is obvious that $|\nabla \xi| \leqslant 2$. Employing (3.2), (3.3), we get the inequalities

$$
J_{2} \leqslant \sum_{\alpha=1}^{n} \int_{\Omega(r)} \xi^{p} T_{\alpha}\left(\frac{C_{5}}{\xi}\right) d x \leqslant C_{6} \int_{\Omega(r) \cap\left\{x \mid C_{5} / \xi(x)<1\right\}} \xi^{p-q_{n}} d x+C_{7} \int_{\Omega(r) \cap\left\{x \mid C_{5} / \xi(x) \geqslant 1\right\}} \xi^{p-\tau} d x .
$$

As a result we have

$$
J_{2} \leqslant C_{8} r^{n-q_{n}+p}, \quad r \geqslant 1, r>r_{0} .
$$

It is obvious that $\xi(x) \geqslant r-r_{0}$ as $|x| \leqslant r_{0}$ and this is why by (4.6), (4.8) we get the inequality

$$
\|\mathbf{B}(u)\|_{1, \Omega\left(r_{0}\right)} \leqslant C_{9}\left(\frac{r}{r-r_{0}}\right)^{p}\left(\|\Psi+\psi\|_{1, \Omega(r)}+r^{n-q_{n}}\right) .
$$

Letting $r_{0}=r / 2$ in 4.9 , we arrive at estimate (2.10).

Corollary 1. Assume that conditions (0.3) - 0.5 hold with $\psi=\Psi=0$ in $\Omega$ and conditions (2.6) - 2.9). Then a generalized solution $u(x)$ to problem $(0.1), 0.2$ vanishes in $\Omega$.

Indeed, letting $\psi(x)=\Psi(x)=0, x \in \Omega$, in (4.9) letting $r$ tend to infinity, we obtain that $\|\mathbf{B}(u)\|_{1, \Omega\left(r_{0}\right)}=0$ for each $r_{0}>0$. It follows that $B_{0}(u)=0$ in $\Omega$ and hence $u=0$ in $\Omega$.

\section{Proof of Theorem 3}

We fix $r \geqslant \max \left(2 r_{1}, r_{2}, 32 D\right)$ ( $r_{1}$ is from condition (2.12), while $r_{2}$ will be detemined below). Let $\theta(x), x>0$, be an absolutely continuous function equallting to one as $x \leqslant r / 2$, vanishing as $x \geqslant 2 r$, being linear as $x \in[r, 2 r]$ and satisfying the equation

$$
\theta^{\prime}(x)=-\delta \theta(x), \quad x \in(r / 2, r),
$$

the constant $\delta$ will be determined later. Solving this equation, we find

$$
\theta(x)=\exp (-\delta(x-r / 2)), \quad x \in(r / 2, r),
$$


then

$$
\theta^{\prime}(x)=\frac{\theta(r)}{r}=\frac{1}{r} \exp (-\delta r / 2), \quad x \in(r, 2 r) .
$$

Letting $\xi(x)=\theta^{p}(|x|), p \geqslant \tau$, in (4.1) and applying (5.1), (5.2), we obtain

$$
\begin{aligned}
& \int_{\Omega} \theta^{p}\left(\sum_{\alpha=1}^{n} a_{\alpha}(x, u, \nabla u) u_{x_{\alpha}}+a_{0}(x, u, \nabla u) u\right) d x \leqslant p \sum_{\alpha=1}^{n} \int_{\Omega(r) \backslash \Omega(r / 2)}\left|u \| a_{\alpha}(x, u, \nabla u)\right| \delta \theta^{p} d x \\
& \quad+p \sum_{\alpha=1}^{n} \int_{\Omega(2 r) \backslash \Omega(r)} \theta^{p-1}|u|\left|a_{\alpha}(x, u, \nabla u)\right| \frac{\theta(r)}{r} d x=p I_{1}+p I_{2} .
\end{aligned}
$$

Employing (1.1), be means of (0.4), 2.11) we estimate the first integral $\left(\varepsilon_{1} \in(0,1)\right)$ :

$$
\begin{aligned}
I_{1} & \leqslant \sum_{\alpha=1}^{n} \int_{\Omega(r) \backslash \Omega(r / 2)} \theta^{p}\left(\bar{B}\left(\varepsilon_{1} a_{\alpha}(x, u, \nabla u)\right)+B\left(u \frac{\delta}{\varepsilon_{1}}\right)\right) d x \\
& \leqslant \int_{\Omega(r) \backslash \Omega(r / 2)} \theta^{p}\left(\varepsilon_{1} \widehat{A} \sum_{\alpha=1}^{n} B\left(u_{x_{\alpha}}\right)+\Psi\right) d x+I_{12}, \\
I_{12} & =\int_{\Omega(r) \backslash \Omega(r / 2)} \theta^{p} B\left(u \frac{\delta}{\varepsilon_{1}}\right) d x .
\end{aligned}
$$

We choose $\varepsilon_{1} \leqslant \frac{\bar{a}}{8 \widehat{A p} p}$ and $\delta$ so that $\delta \leqslant \varepsilon_{1}$.

Thanks to the inclusion $\gamma(\rho) \subset \Sigma_{\rho, 2 d(\rho)}$, the inequality

$$
I_{12} \leqslant \frac{\delta}{\varepsilon_{1}} \int_{r / 2}^{r} \theta^{p}(\rho) \int_{\Sigma_{\rho, 2 d(\rho)}} B(u) d S d \rho
$$

holds true for a function $u(x) \in C_{0}^{\infty}(\Omega)$.

Employing inequality (3.4) and condition $(2.12)$ as well as $(1.2)$, we get

$$
I_{12} \leqslant \frac{\delta}{\varepsilon_{1}} c \int_{r / 2}^{r} \theta^{p}(\rho) \int_{\Sigma_{\rho, 2 d(\rho)}} B(2 d(\rho)|\nabla u|) d S d \rho \leqslant C_{1} \frac{\delta}{\varepsilon_{1}} \int_{r / 2}^{r} \theta^{p}(\rho) \int_{\gamma(\rho)} B(|\nabla u|) d S d \rho .
$$

By 1.3 we obtain the inequality

$$
I_{12} \leqslant C_{2} \frac{\delta}{\varepsilon_{1}} \sum_{\alpha=1}^{n} \int_{\Omega(r) \backslash \Omega(r / 2)} \theta^{p}(|x|) B\left(u_{x_{\alpha}}\right) d x .
$$

Employing Lemma 2 and passing to a limit, we get inequality (5.5) for a function $u(x) \in$ $\stackrel{\circ}{W}_{\mathbf{B}, \text { loc }}^{1}(\Omega)$. Combining (5.4), (5.5), choosing $\delta C_{2} \leqslant \varepsilon_{1} \frac{\bar{a}}{8 p}$, we obtain

$$
I_{1} \leqslant \frac{\bar{a}}{4 p} \int_{\Omega(r) \backslash \Omega(r / 2)} \theta^{p} \mathbf{B}(u) d x+\|\Psi\|_{1, \Omega(r) \backslash \Omega(r / 2)} .
$$

Let us estimate the integral $I_{2}$. Employing (1.1), for $\varepsilon_{2} \in(0,1)$ we get

$$
I_{2} \leqslant \sum_{\alpha=1}^{n} \int_{\Omega(2 r) \backslash \Omega(r)} \theta^{p} \bar{B}\left(\varepsilon_{2} a_{\alpha}(x, u, \nabla u)\right) d x+n \int_{\Omega(2 r) \backslash \Omega(r)} \theta^{p} B\left(\frac{u}{\varepsilon_{2}} \frac{\theta(r)}{r \theta(|x|)}\right) d x=I_{21}+I_{22} .
$$


Let us estimate the integral $I_{22}$. Thanks to relations (3.1), we can represent the $N$-function $B_{0}(z)=B(M(z))$ as a composition of two $N$-functions $M(z), B(z)$. Employing (1.1), (1.3), we obtain

$$
\begin{aligned}
I_{22} \leqslant & \int_{\Omega(2 r) \backslash \Omega(r)} \theta^{p} B\left\{M\left(\varepsilon_{2} u\right)+\bar{M}\left(\frac{1}{\varepsilon_{2}^{2}} \frac{\theta(r)}{r \theta(|x|)}\right)\right\} d x \\
& \leqslant n \int_{\Omega(2 r) \backslash \Omega(r)} \theta^{p}\left(\varepsilon_{2} C_{3} B_{0}(u)+C_{3} B\left(\bar{M}\left(\frac{1}{\varepsilon_{2}^{2}} \frac{\theta(r)}{r \theta(|x|)}\right)\right)\right) d x \\
& =C_{3} n\left(\varepsilon_{\Omega(2 r) \backslash \Omega(r)}^{\varepsilon_{2}} \theta^{p} B_{0}(u) d x+I_{3}\right),
\end{aligned}
$$

where

$$
I_{3}=\int_{\Omega(2 r) \backslash \Omega(r)} \theta^{p} T\left(\frac{1}{\varepsilon_{2}^{2}} \frac{\theta(r)}{r \theta(|x|)}\right) d x, \quad T(z)=B(\bar{M}(z)) .
$$

Combining (5.7), (5.8) and employing condition (0.4), we get

$$
\begin{aligned}
I_{2} \leqslant & \varepsilon_{2} \int_{\Omega(2 r) \backslash \Omega(r)} \theta^{p}\left(\widehat{A} \sum_{\alpha=1}^{n} B_{\alpha}\left(u_{x_{\alpha}}\right)+\left(n C_{3}+\widehat{A}\right) B_{0}(u)\right) d x \\
& +\int_{\Omega(2 r) \backslash \Omega(r)} \theta^{p} \Psi d x+n C_{3} I_{3} \leqslant \varepsilon_{2} C_{4} \int_{\Omega(2 r) \backslash \Omega(r)} \theta^{p} \mathbf{B}(u) d x+\int_{\Omega(2 r) \backslash \Omega(r)} \Psi d x+C_{4} I_{3} .
\end{aligned}
$$

We choose $\varepsilon_{2} \leqslant \frac{\bar{a}}{4 C_{4} p}$ to obtain

$$
I_{2} \leqslant \frac{\bar{a}}{2} \int_{\Omega(2 r) \backslash \Omega(r)} \theta^{p} \mathbf{B}(u) d x+\int_{\Omega(2 r) \backslash \Omega(r)} \Psi d x+C_{4} I_{3} .
$$

Substituting estimates (5.6), (5.11) into (5.3) and employing condition (0.3), we get

$$
\|\mathbf{B}(u)\|_{1, \Omega(r / 2)} \leqslant C_{5} \int_{\Omega(2 r)}\{\Psi+\psi\} d x+C_{5} I_{3} .
$$

Let us estimate the integral $I_{3}$. We let $r_{2}=1 / \varepsilon_{2}^{2}$, then as $r \geqslant r_{2}$, due to the convexity of the function $T(z)$, the inequality

$$
I_{3} \leqslant \frac{r_{2}}{r} \int_{\Omega(2 r) \backslash \Omega(r)} \theta^{p} T\left(\frac{\theta(r)}{\theta(|x|)}\right) d x
$$

holds true. As $|x| \in(r, 2 r)$, the inequality $\theta(|x|) \leqslant \theta(r)$ is satisfied and applying Lemma 5, we obtain the estimate

$$
I_{3} \leqslant \frac{C_{6}}{r} \int_{\Omega(2 r) \backslash \Omega(r)} \theta^{p-\tau}(|x|) \theta^{\tau}(r) d x \leqslant C_{7} r^{n-1} \exp (-\delta p r / 2) .
$$

Combining (5.12), 5.13), we get 2.13). 


\section{EXAMPLES}

Example 1. Let $n=3, p_{1}=11 / 3, p_{2}=11 / 4, p_{3}=11 / 5$,

$$
B_{\alpha}(z)= \begin{cases}|z|^{p_{\alpha}}, & |z|<1 \\ |z|^{p_{\alpha}-1}(\ln |z|+1), & |z| \geqslant 1, \quad \alpha=1,2,3 .\end{cases}
$$

Since $|z|^{p_{\alpha}-1} \leqslant B_{\alpha}(z) \leqslant|z|^{p_{\alpha}}$ as $|z| \geqslant 1$, then $t^{1 /\left(p_{\alpha}-1\right)} \geqslant B_{\alpha}^{-1}(t) \geqslant t^{1 / p_{\alpha}}$ as $t \geqslant 1, \alpha=1,2,3$. It follows that

$$
\begin{aligned}
& h(t)=t^{1 / 33}, 0<t<1, \quad \int_{0}^{1} t^{-1} h(t) d t<\infty, \\
& t^{131 / 504} \geqslant h(t) \geqslant t^{1 / 33}, t \geqslant 1, \quad \int_{1}^{\infty} t^{-1} h(t) d t=\infty,
\end{aligned}
$$

and hence, we can define the functions $\left(B^{*}\right)^{-1}(t), B^{*}(z)$ and to satisfy the relations

$$
\begin{aligned}
& \left(B^{*}\right)^{-1}(t)=33 t^{1 / 33}, 0<t<1, \quad 504 / 131 t^{131 / 504} \geqslant\left(B^{*}\right)^{-1}(t) \geqslant 33 t^{1 / 33}, t \geqslant 1, \\
& B^{*}(z)=(|z| / 33)^{33},|z|<33, \quad(131 / 504|z|)^{504 / 131} \leqslant B^{*}(|z|) \leqslant(|z| / 33)^{33},|z| \geqslant 33 .
\end{aligned}
$$

We take $B_{0}(z)=|z|^{42 / 11}$. Such choise of the functions $B_{\alpha}(z), \alpha=0,1,2,3$, ensures conditions (2.1), (2.6).

We consider the functions $a_{0}(x, z)=|z|^{20 / 11} z+f_{0}(x)$,

$$
a_{\alpha}(x, z)=f_{\alpha}(x)+B_{\alpha}^{\prime}(z)=f_{\alpha}(x)+\left\{\begin{array}{ll}
p_{\alpha}|z|^{p_{\alpha}-2} z, & |z|<1 \\
|z|^{p_{\alpha}-3} z\left(\left(p_{\alpha}-1\right) \ln |z|+p_{\alpha}\right), & |z| \geqslant 1
\end{array},\right.
$$

$f_{\alpha} \in L_{\bar{B}_{\alpha}, \text { loc }}(\bar{\Omega}), \quad \alpha=0,1,2,3$. According to Lemma 4, conditions $(0.3)-(0.5)$ are satisfied. Thus, by Theorem 1, there exists a generalized solution to problem (0.1), (0.2).

Since $1 / p_{1}+1 / p_{2}+1 / p_{3}=12 / 11>1, q_{3}=\frac{p_{0} p_{3}}{p_{0}-p_{3}}=462 / 89>3$, conditions (2.8), (2.9) are satisfied as well. According to Theorem 2, generalized solution to problem (0.1), (0.2) obeys the estimate

$$
\|\mathbf{B}(u)\|_{1, \Omega(r / 2)} \leqslant M\left(r^{-195 / 89}+\|\psi+\Psi\|_{1, \Omega(r)}\right), \quad r \geqslant 1 .
$$

Example 2. Let $n>2,2<p<n$,

$$
B(z)= \begin{cases}|z|^{p-1}\left(-\ln |z|+\frac{p+1}{p-1}\right), & |z|<1 \\ \frac{2}{p-1}+|z|^{p-1}(\ln |z|+1), & |z| \geqslant 1\end{cases}
$$

Since $B(z) \geqslant \frac{p+1}{p-1}|z|^{p-1}$ as $|z|<1$, then $B^{-1}(t) \leqslant\left(\frac{p-1}{p+1} t\right)^{1 /(p-1)}$ as $0<t<\frac{p+1}{p-1}$. Moreover, $|z|^{p-1} \leqslant B(z) \leqslant \frac{p+1}{p-1}|z|^{p}$ as $|z| \geqslant 1$, and this is why $\left(\frac{p-1}{p+1} t\right)^{1 / p} \leqslant B^{-1}(t) \leqslant t^{1 /(p-1)}$ as $t \geqslant \frac{p+1}{p-1}$. It follows that

$$
\begin{aligned}
& h(t) \leqslant C_{1} t^{\frac{n-p+1}{n(p-1)}}, \quad 0<t<\frac{p+1}{p-1}, \quad \int_{0}^{1} t^{-1} h(t) d t<\infty, \\
& C_{2} t^{\frac{n-p}{n p}} \leqslant h(t) \leqslant t^{\frac{n-p+1}{n(p-1)}}, \quad t \geqslant \frac{p+1}{p-1}, \quad \int_{1}^{\infty} t^{-1} h(t) d t=\infty,
\end{aligned}
$$

and we can define functions $\left(B^{*}\right)^{-1}(t), B^{*}(z)$ and to satisfy the inequalities

$$
\begin{aligned}
& C_{3}|z|^{\frac{n-p}{n p}} \leqslant\left(B^{*}\right)^{-1}(z) \leqslant C_{4}|z|^{\frac{n-p+1}{n(p-1)}},|z| \geqslant \frac{p+1}{p-1}, \\
& C_{5}|z|^{\frac{n(p-1)}{n-p+1}} \leqslant B^{*}(z) \leqslant C_{6}|z|^{\frac{n p}{n-p}},|z| \geqslant C_{7} .
\end{aligned}
$$


We take $p_{0}=\frac{n(p-1)}{n-p+1}$,

$$
B_{0}(z)= \begin{cases}|z|^{p_{0}-1}\left(-\ln |z|+\frac{p_{0}+1}{p_{0}-1}\right), & |z|<1 \\ \frac{2}{p_{0}-1}+|z|^{p_{0}-1}(\ln |z|+1), & |z| \geqslant 1 .\end{cases}
$$

Such choice of the functions $B_{0}(z), B(z)$ as $p>(1+\sqrt{1+4 n}) / 2$ ensures conditions (2.1), (2.6).

We consider the functions

$$
a_{\alpha}(x, z)=f_{\alpha}(x)+B^{\prime}(z)=f_{\alpha}(x)+|z|^{p-3} z((p-1)|\ln | z||+p), \alpha=1, \ldots, n,
$$

$a_{0}(x, z)=f_{0}(x)+B_{0}^{\prime}(z)=|z|^{p_{0}-3} z\left(\left(p_{0}-1\right)|\ln | z||+p_{0}\right)$. According to Lemma 4, conditions (0.3)- (0.5) are satisfied. Thus, by Theorem 1, there exist a generalized solution to problem (0.1), (0.2).

According to Theorem 3, a generalized solution to problem $0.1,0.2$ in the domains satisfying condition $(2.12)$ obeys the estimate

$$
\|\mathbf{B}(u)\|_{1, \Omega(r / 2)} \leqslant \mathcal{M}_{2}\left(\exp (-\kappa r) r^{n-1}+\|\psi+\Psi\|_{1, \Omega(2 r)}\right), \quad r \geqslant r_{0} .
$$

\section{BIBLIOGRAPHY}

1. T. Donaldson. Nonlinear elliptic boundari value problems in Orlicz-Sobolev spaces // J. Diff. Equat. 10:3, 507-528 (1971).

2. V.S. Klimov. Imbedding theorems for Orlicz spaces and their applications to boundary value problems // Sibir. Matem. Zhurn. 13:2, 334-348 (1972). [Sib. Math. J. 13:2, 231-240 (1972).]

3. A. Fougeres. Opérateurs elliptiques du calcul des variations à coefficients tres fortement non linéaires // C.R. Acad. Sci. Paris Ser. A. 274, 763-766 (1972).

4. J.P. Gossez. Nonlinear elliptic boundary value problems for equations with rapidly (or slowly) increasing coefficients // Trans. Amer. Math. Soc. 190, 163-206 (1974).

5. P. Gwiazda, P. Wittbold, A. Wróblewska, A. Zimmermann. Renormalized solutions of nonlinear elliptic problems in generalized Orlicz spaces. Nonlinear Anal.: Theory Methods Appl. 129, 1-36 (2015).

6. R.Kh. Karimov, L.M. Kozhevnikova. Behavior on infinity of decision quasilinear elliptical equations in unbounded domain // Ufimskij Matem. Zhurn. 2:2, 53-66 (2010). (in Russian).

7. L. M. Kozhevnikova, A. A. Khadzhi. Solutions of anisotropic elliptic equations in unbounded domains // Vestn. Samar. Gos. Tekhn. Univ. Ser. Fiz.-Mat. Nauki. 1(30):90-96 (2013) (in Russian).

8. A.L. Gladkov. The Dirichlet problem for quasilinear degenerating elliptic equations in unbounded domains // Differ. Uravn. 29:2, 267-273 (1993). [Differ. Equat. 29:2, 217-223 (1993).]

9. H. Brezis. Semilinear equations in $R_{N}$ without condition at infnity // Appl. Math. Optim. 12:3, 271-282 (1984).

10. F. Bernis. Elliptic and parabolic semilinear problems without conditions at infnity // Arch. Rational Mech. Anal. 106:3, 217-241 (1989).

11. J.I. Diaz, O.A. Oleinik. Nonlinear elliptic boundary-value problems in unbounded domains and the asymptotic behaviour of its solution // C.R. Acad. Sci. Paris. Ser. I Math. 315:1, 787-792 (1992).

12. M. Bokalo, O. Domanska. On well-posedness of boundary problems for elliptic equations in general anisotropic Lebesgue-Sobolev spaces // Mat. Stud. 28:1, 77-91 (2007).

13. L.M. Kozhevnikova, A.A. Khadzhi. Existence of solutions of anisotropic elliptic equations with nonpolynomial nonlinearities in unbounded domains // Matem. Sborn. 206:8, 99-126 (2015). [Sb. Math. 206:8, 1123-1149 (2015).]

14. L.M. Kozhevnikova, A.A. Khadzhi. Uniqueness of solutions to anisotropic elliptic equations with nonpower nonlinearities in unbounded domains // Probl. Matem. Anal. 85, 153-163 (2016). [J. Math. Sci. 219:1, 160-171 (2016).]

15. A. Krasnosel'skiy, Ya.B. Rutitskiy. Convex functions and Orlicz spaces. Fizmatlit, Moscow (1958). [P. Noordhoff Ltd., Groningen, (1961).] 
16. A.G. Korolev. Embedding theorems for anisotropic SobolevOrlicz spaces // Vestn. Mosk. Univ. Ser 1. 1, 32-37 (1983). [Mosc. Univ. Math. Bull., 38:1, 37-42 (1983).]

17. L.M. Kozhevnikova, A.A. Khadzhi. On solutions of elliptic equations with nonpower nonlinearities in unbounded domains // Vestn. Samar. Gos. Tekhn. Univ. Ser. Fiz.-Mat. Nauki. 19:1, 44-62 (2015) (in Russian).

18. L.M. Kozhevnikova, R.Kh. Karimov, A.A. Khadzhi. On behavior of solutions to elliptic equations with non-power nonlinearities in unbounded domains // Aktual'nye problemy gumanitarnych i estestevennych nauk. 9, 3-17 (2015) (in Russian).

19. A.A. Khadzhi. On Fridrichs type inequality // Nauchno-tekhnicheskii vestnik Povolzh'ya. 9, 30-33 (2015) (in Russian).

Ruslan Khalikovich Karimov,

Sterlitamak branch of Bahkir State Univeristy,

Lenin av. 37,

453103, Sterlitamak, Russia

E-mail: ruslan7k7@mail.ru

Larisa Mikhailovna Kozhevnikova,

Sterlitamak branch of Bahkir State Univeristy,

Lenin av. 37,

453103, Sterlitamak, Russia

Elabuga Institute of Kazan Federal University,

Kazanskaya str. 89, 423600, Elabuga, Russia

E-mail: kosul@mail.ru

Anna Alexandrovna Khadzhi,

Tyumen State University,

Volodarskii str. 6, Tyumen,

625003, Tyumen, Russia

E-mail: anna_5955@mail.ru 